\title{
A Ring-Cavity Diode Laser
}

\author{
Jianing Han*, Lindsay Hutcherson, Kaori Munekane, Steven Shettlesworth
}

Physics Department, University of South Alabama, Mobile, AL, USA

Email: *jhan@southalabama.edu

How to cite this paper: Han, J.N., Hutcherson, L., Munekane, K. and Shettlesworth, S. (2020) A Ring-Cavity Diode Laser. Journal of Modern Physics, 11, 1236-1244.

https://doi.org/10.4236/jmp.2020.118077

Received: October 17, 2019

Accepted: August 22, 2020

Published: August 25, 2020

Copyright (C) 2020 by author(s) and Scientific Research Publishing Inc.

This work is licensed under the Creative Commons Attribution International License $(\mathrm{CC}$ BY 4.0).

http://creativecommons.org/licenses/by/4.0/

\section{Open Access}

\begin{abstract}
In this article, we report on a ring-cavity diode laser configuration, which has more features compared to the standing-wave externalcavity diode lasers. First, a transmission grating will be used, and the ring-cavity laser output will be the zero-order output from the transmission grating. Therefore, unlike the standing-wave external cavity diode lasers, the output direction of this ring-cavity laser does not depend on the laser's frequency. Second, since it is a ringcavity laser, it can avoid the local heating caused by the antinodes of the standing-wave lasers. Moreover, because this ring-cavity will allow the laser beam to pass the transmission grating two times, it is expected that this will ultimately result in a narrower linewidth compared to the external-cavity standing-wave lasers with the same cavity length.
\end{abstract}

\section{Keywords}

PACS Numbers: 07.05.Fb, 42.15.Eq, 42.55.Px

\section{Introduction}

Van der Waals interactions are commonly known as attractive interactions, which is only true for ground-state atoms. Recent studies [1-4] show that repulsive van der Waals interactions can exist in excited states. Therefore, Rydberg atoms are essential for doing repulsive van der Waals interactions. The Rydberg excitation is often done by lasers, so lasers [5-7] are the key tools to study such interactions. Compared to dye lasers, an external-cavity diode laser has a much narrower bandwidth (about $1 \mathrm{MHz}$ ), and recent experiments are done by external-cavity diode lasers [1-4]. In this article, we will focus on external-cavity diode lasers. The cavity of diode lasers is made of the front and back surface of the diode. To improve the tunability of this type of laser and achieve a narrower linewidth, an external-cavity is often installed (Ref. [8] and references therein).

In this article, we report on a ring-cavity diode laser. This experiment is motivated by the following three facts: first, the widely used standing-wave external-cavity lasers $[8,9]$ form standing waves between 
a reflective grating and the back surface of the laser diode. In other words, a reflective grating and the back surface of the laser diode form the external cavity of the standing-wave diode lasers. The first order of the reflective grating is directly sent back to the laser diode, and the zero-order of this reflective grating is the output of the standing-wave external-cavity laser. Therefore, the laser's wavelength or frequency depends on the output beam's angle. In other words, once the laser's frequency is changed, the output direction will also change. If the laser frequency needs to be constantly adjusted, this will cause a lot of downstream alignment issues. This is an important consideration in Rydberg physics. The output of this ring-cavity laser proposed is the zero-order of a transmission grating instead of a reflective grating. Therefore, the output direction will not be affected when the frequency is changed, or the output direction does not depend on the laser's frequency. Similar to the Littman configurations [10,11], the configuration presented in this article applies to diode lasers. This is crucial for studying repulsive van der Waals interactions, which only exist in excited states [1-4]. Second, this type of laser is expected to produce a narrower linewidth compared to the standing-wave external-cavity diode lasers. The linewidth of the ring-cavity laser can be reduced by allowing the laser beam within the cavity to pass the transmission grating two times. Third, the ring-cavity laser [12] can avoid the local heating caused by the antinodes of the standing waves. A standing wave is formed by two counter-propagating waves. Within a standing-wave cavity, there are nodes and anti-nodes along the cavity, or the intensity variation, which will result in non-uniform heating, or local heating, of the laser medium or a diode in this case. The ring-cavity configuration can avoid local heating [13]. The waves in a ring-cavity are propagating waves, so no nodes or antinodes will be formed; the heating will be uniform. The laser intensity stability of the standing-wave external-cavity lasers is partially caused by the local heating from the antinodes of the standing wave in the laser diode, especially at high intensities, which will limit the ability of creating bigger MOTs (Magneto-Optical Traps).

This ring-cavity laser has many potential applications. Related to earlier discussions, lasers can be used to excite atoms from ground states to excited states, or Rydberg states $[14,15]$. In addition, the standing-wave external-cavity diode lasers $[8,9,16]$ are the workhorses in the cold atom field. It can be used for laser cooling and trapping. Since the output is the zero-order output of a transmission grating, this will make the optics adjustment a lot easier. This system can be used to study the amplification of the ring-cavity laser using tapered amplifiers or other types of lasers [17]. In addition, this system can be used as a frequency comb for high precision measurement [18] and laser spectroscopy, etc.

This paper is arranged in the following way: the experiment is introduced in the next paragraph, which is followed by the tunability measurement. The output power from the ring-cavity laser is then discussed and analyzed. A conclusion is given at the end.

\section{Experiment}

In this experiment, we use a PLT5 488 diode, manufactured by OSRAM Opto Semiconductors, mounted in a temperature and current controller kit purchased from Thorlabs, which includes the laser mount. Figure 1 shows the schematic diagram of this ring-cavity laser. The 
grey line is the laser beam pass. The beam coming out of the laser first passes through a grating. In order to avoid the zero-order back reflection from the grating, it is recommended to tilt the angle of the grating slightly, so the grating is not parallel to the laser diode surface. After the grating, the beam diffracts. The first order will be reflected by mirror 1 , then reflected again by mirror 2 . The reflected beam from mirror 2 is sent back to the laser along the opposite direction of the -1 order direction of the grating. In other words, the laser beam passes the grating again. As described earlier, this laser can produce a narrower linewidth than the standing wave lasers with the same cycle length by allowing the laser beam to pass the grating two times. Here the cycle length is the total length for the laser beam to pass one cycle. For example, the cycle length of a ring cavity is the same as the cavity length $\left(2 l_{1}+l_{2}+l_{3}+l_{4}\right)$, while the cycle length is $2 l^{\prime}$ for a standing wave cavity, where $l^{\prime}$ is the distance between the two standing wave cavity mirrors. After the final reflected beam enters the grating, the laser beam passes the laser diode, reaches the back surface of the diode, and completes the ring-cavity. The zero-order is the output of the ring-cavity laser. The laser beam is collimated to $0.6 \mathrm{~mm}$ in diameter, which is measured at $\mathrm{I}=90$ $\mathrm{mA}$ using the knife-edge method, a knife blade mounted on a translation stage. The grating used in this particular experiment is a 1000 line per millimeter transmission grating. The first-order diffraction efficiency, (the output power in the first-order diffracted beam)/(the output power without the grating), is about $35.4 \%$, and the secondorder diffraction efficiency is about $5.33 \%$. The third and above order diffraction efficiencies are zero. Both diffraction efficiencies are measured at $90 \mathrm{~mA}$, and the total output power of the diode before the grating at this current is about $52.5 \mathrm{~mW}$. The mirrors are aluminumcoated mirrors. The reason that aluminum-coated mirrors are used is that those mirrors have higher reflectivity in the wavelength range that we studied and low transmissivity. The total cavity length of this ring-cavity, $2 l_{1}+l_{2}+l_{3}+l_{4}$, is about $16 \mathrm{~cm}$. The linewidth measurement was done through a Fabry-Perot interferometer (model number: SA200-3B) borrowed from Thorlabs. The free spectrum range of this Fabry-Perot interferometer is $1.5 \mathrm{GHz}$, and the resolution is $7.5 \mathrm{MHz}$. We connect the Fabry-Perot interferometer output to an oscilloscope. The oscilloscope is then connected to a GPIB board, which is connected with a computer. All the oscilloscope traces are recorded on a computer through this GPIB board.

\section{Result and Discussion}

The $Q$ factor of this cavity can be calculated by the following equation $[5]$ :

$$
Q=\frac{v_{0}}{v_{F}} F
$$

where $v_{0}$ is the resonant frequency, $v_{0}=\frac{c}{488 n m}$ in our case, and $c$ is the speed of light. $v_{F}$ is the cavity frequency difference, $v_{F}=\frac{c}{2 l_{1}+l_{2}+l_{3}+l_{4}}$ in this cavity (Figure 1). In addition, $F$ is the Finesse of the cavity shown in Figure 1:

$$
F=\frac{\pi r \sqrt{r_{1}}}{1-r_{1} r^{2}}
$$




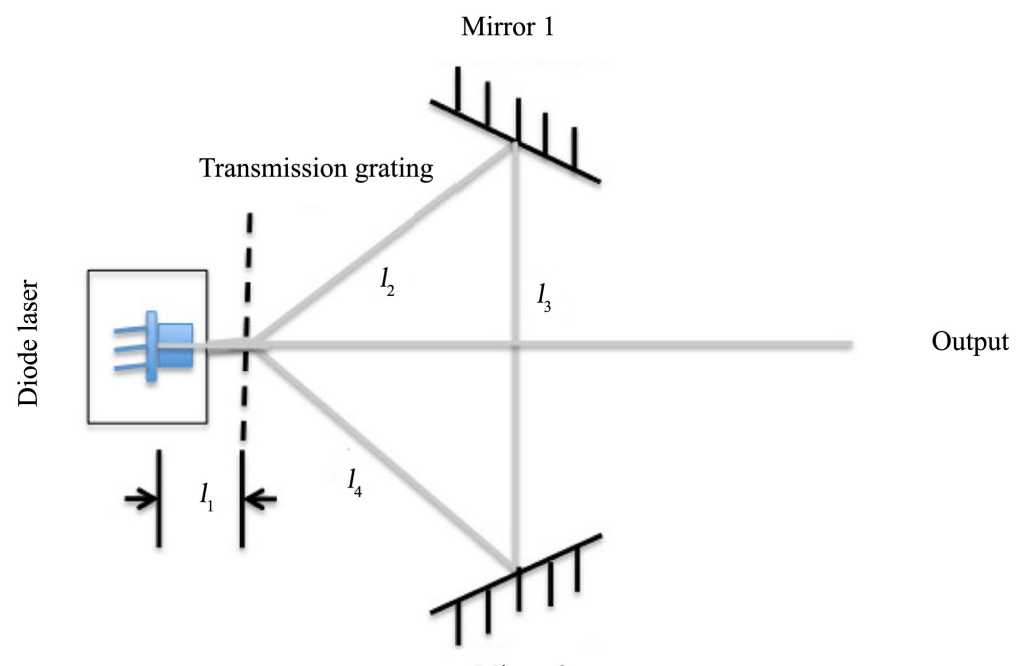

Figure 1. The schematic diagram of the ring-cavity laser. $l_{1}$ is the distance between the back surface of the diode laser and the grating. $l_{2}$ is the distance between the grating and mirror $1 . l_{3}$ is the distance between mirror 1 and mirror 2. $l_{4}$ is the distance between mirror 2 and the grating.

where $r_{1}$ and $r$ are the amplitude attenuation factor introduced by the reflection from the back side of the diode and the reflection of one of the mirrors. The absorption or energy loss by the grating and the medium is also included in these terms. $r_{1}$ is much smaller than $r$ in our case, since $r_{1}$ includes the energy loss caused by the reflection from the front side of the diode. If $r \approx 80 \%$ and $r_{1} \approx 50 \%$, the $Q$ factor of this $488 \mathrm{~nm}$ external laser cavity shown in Figure 1 is about $8.6 \times 10^{5}$.

One of the features of this ring-cavity laser is that the frequency is tunable. The way to understand this is that the cavity selects a certain frequency, which is used as a seed to be sent back to the cavity. This seed beam will pull the frequency of the zero-order beam to this selected frequency. Therefore, by changing the cavity length, the laser frequency can be changed. To show the tunability, we first show that we are able to measure the laser's relative output frequency. We send the ring cavity laser output to a Fabry-Perot interferometer. Figure 2 shows two oscilloscope traces taken from the Fabry-Perot interferometer. To reduce the power broadening and laser mode hopping at high intensity, we set the laser diode current at a low current. The laser current in Figure 2 and Figure 3 is below $55 \mathrm{~mA}$. Both traces are taken by directly connecting the photodiode of the Fabry-Perot interferometer to an oscilloscope. A time-dependent voltage controlling the Fabry-Perot cavity length is added to the Fabry-Perot cavity. As the voltage changes, the light intensity as a function of time collected by the photodiode is sent to the oscilloscope. The left figure, Figure 2(a), is a long scan. The horizontal axis is converted from the original time to frequency by setting the frequency difference between the two peaks as $1.5 \mathrm{GHz}$, the free-spectrum range of this Fabry-Perot interferometer. The off-set frequency can be calculated by

$$
f=\frac{c}{\lambda},
$$

where $c$ is the speed of light, and $\lambda=488 \mathrm{~nm}$ is the wavelength of this laser. The off-set frequency is calculated to be about $6.15 \times 10^{5} \mathrm{GHz}$. 


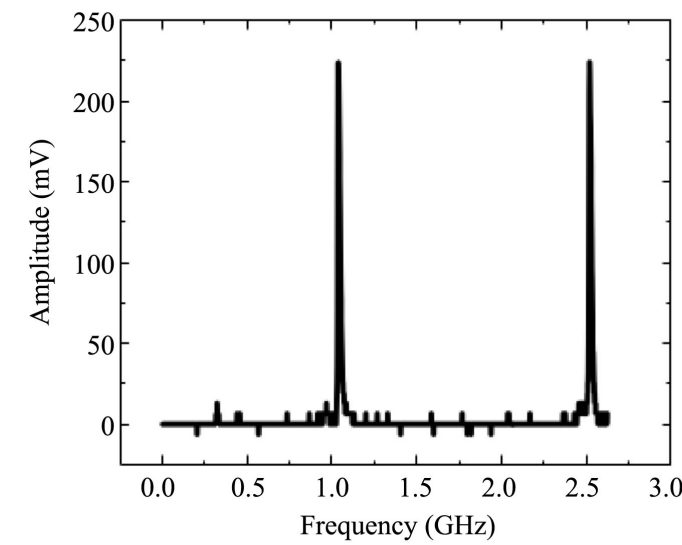

(a)

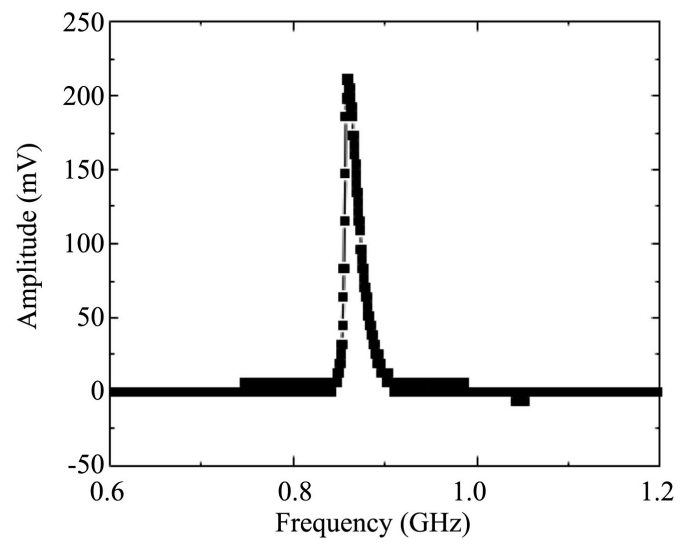

(b)

Figure 2. (a) is a long scan of the trace; (b) The detail of a single peak.

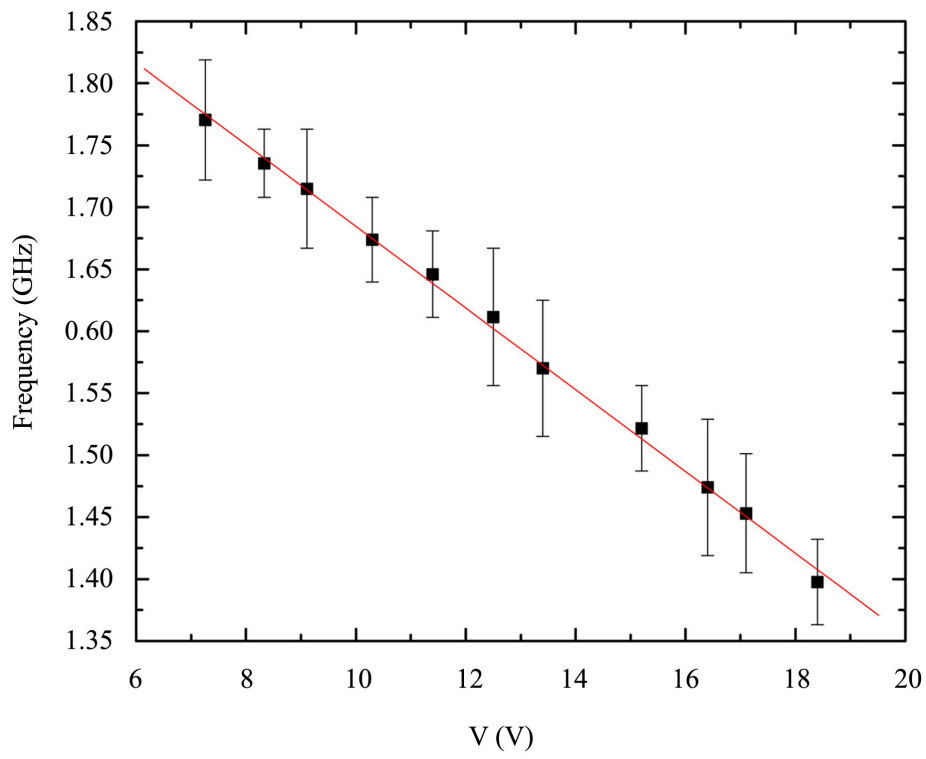

Figure 3. The relative frequency of the laser as a function of the voltage on the piezoelectric actuator. The red solid line is the linear fit of the experimental data.

To show the details of one of the peaks, we expand the horizontal axis of the oscilloscope and collect a trace for one of the peaks, as shown in Figure 2(b). The FWHM (full width at half maximum) of the trace shown in Figure 2(b) is about $17 \mathrm{MHz}$, which is close to the resolution of this Fabry-Perot interferometer.

The frequency of this type of laser is adjustable. To change the output frequency of this ring-cavity laser, we change the angle of mirror 1 or 2. In this experiment, we use a piezoelectric actuator (PZT) to change the angle of mirror 2. Alternatively, we can change the angle of mirror 1 , and similar results are expected. In this experiment, the piezoelectric actuator is installed in the mirror mount of mirror 2 to control the horizontal direction of the laser beam reflected off of mirror 2. Figure 3 shows the relative laser frequency as a function of the voltage on the piezoelectric actuator, which is used to change the horizontal angle of mirror 2. As we vary the voltage on the piezoelectric actuator, the laser output frequency changes as a function of the 
voltage on the piezoelectric actuator. The data points are obtained in the following way: at each angle or each voltage on the piezoelectric actuator, a few oscilloscope traces are taken. Due to the thermal fluctuation and mechanical vibration of the laser mount, the frequency of the laser jitters. In addition, the power from the laser fluctuates, which will cause the uncertainty of the linewidth of the laser. Therefore, at each voltage, we find the minimum frequency of the few traces taken at a particular voltage and the maximum frequency of those few traces, instead of calculating the average of few frequencies measured at one voltage. We then calculate the average of those two, $\frac{\text { maximum }+ \text { minimum }}{2}$, to get the actual data point shown in Figure 3 as the black solid squares. The error bar is calculated by the difference between the maximum frequency and the average frequency or the difference between the average frequency and the minimum frequency. In other words, the total length of the error bar is (maximum-minimum). The total frequency tuning range of this laser is $488 \mathrm{~nm} \pm 1 \mathrm{~nm}$. The free-running laser linewidth is greater than $1.5 \mathrm{GHz}$.

In addition to the tunability measurement, we also studied the power of this ring-cavity laser. Figure 4 shows the output power comparison between the free-running laser, plotted as red dots, and the output power from the ring-cavity laser, plotted as black squares. The power is measured by a thermopile power meter. This graph shows that the power increases as the current increases, which is what we expected. Comparing those two sets of data, it is also shown that the output power of this ring-cavity laser is much lower than the free-running laser. Specifically, the ring-cavity laser output is only about $20 \%$ of the free-running laser output. In this experiment, since we allow the laser beam to pass the grating two times, we would expect the loss is about twice the power loss from the standing-wave external-cavity laser $[8,9]$. Here we again use the 1000 line/mm grating, and both the first order and second order from the grating are visible, which means that for every ring cycle, the second-order output will cause power loss. This can be avoided by choosing a grating with a higher line density, or the line density greater than 1000 lines per millimeter for a $488 \mathrm{~nm}$ laser. This is something that we would like to explore in the near future. The error bars are based on the maximum power fluctuation from the power meter reading. During the measurement, we noticed that the power typically drifts downward once the current sent to the diode laser is fixed.

We have also tested the mirrors' reflectivity effect on this ring-cavity laser. We changed the reflectivity of the mirrors from $32 \%$ to $80 \%$, and the output difference is less than $3 \%$. In other words, it turns out that by increasing the reflectivity of the cavity mirror, the effect of the mirrors' reflectivity on the output power of the ring-cavity laser is negligible.

Within the ring-cavity laser, we had to increase the current limit of the temperature controller, which controls the temperature of the laser diode, in order to stabilize the temperature of the ring-cavity laser unit. This wasn't the case with the free-running laser. In other words, the free-running laser does not require high current to be stabilized, which indicates that the free-running laser generates less heat than the ring-cavity laser. This may be related to the uniform heating of the laser system or caused by the high intensity within the laser cavity.

Here are a few limitations of this ring-cavity laser. First, the output power is very low. Choosing a grating, which has a greater line 


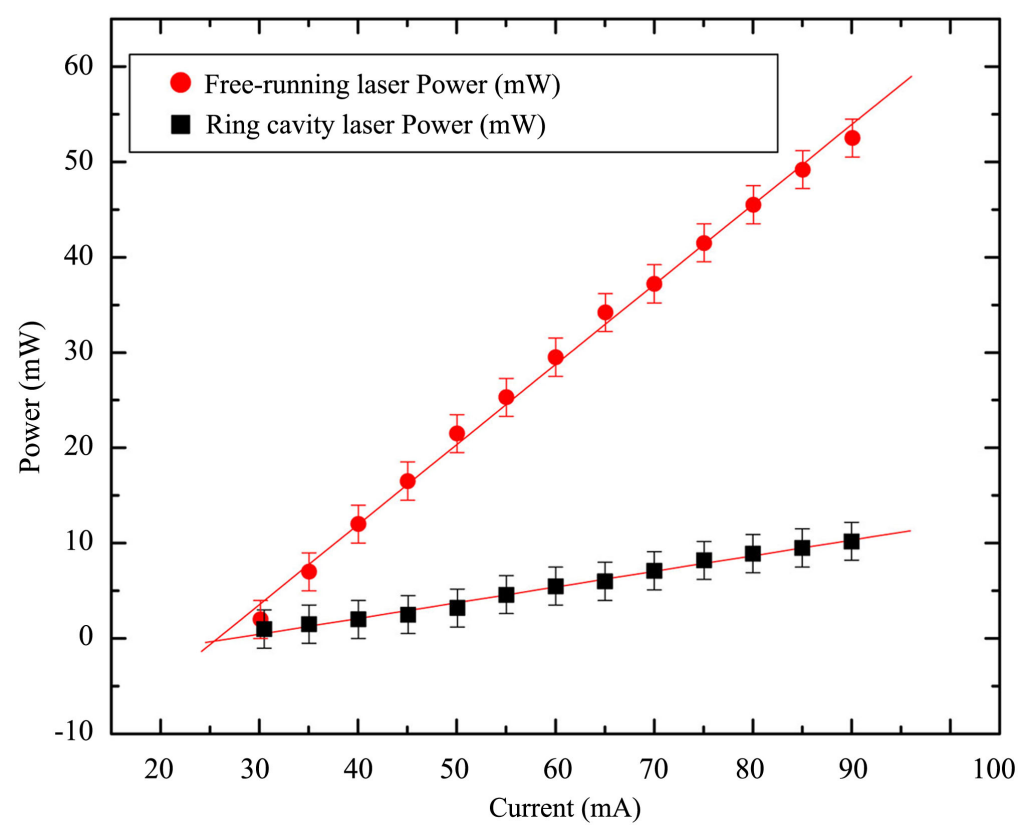

Figure 4. The output power from the free-running laser $(\bullet)$ and the output from the ring-cavity laser $(\boldsymbol{\square})$. The red solid lines are the linear fits of the experimental data.

density, will be helpful. One limitation of diode lasers is the modehopping. Very similar to other diode lasers, this ring-cavity laser also shows mode-hopping, which may be improved by stabilizing the optical table and more stable temperature and current control systems. It is generally observed that as the current increases, the mode hopping rate increases, and the probability of multimode also increases. Changing the internal and external cavity length may help to reduce the mode-hopping rate.

\section{Conclusion}

In conclusion, we have shown a ring-cavity diode laser that is tunable. The frequency can be easily adjusted by adjusting the voltage of a piezoelectric actuator installed in one of the mirror mounts. In addition, this laser output is the zero-order output of a transmission grating; therefore, the direction of this output is frequency independent. This type of laser is very useful for studying excited states atoms, especially for repulsive van der Waals interactions. Moreover, this type of laser is promising to produce narrower linewidth than the standing-wave external-cavity laser with the same cavity length by allowing the laser beam to pass the grating two times.

\section{Acknowledgements}

The authors would like to thank the equipment loan from Thorlabs. This material is based upon work supported by the U. S. Army Research Office (ARO), the travel support from DOE/EPSCOR, and the Air Force Office of Scientific Research (AFOSR). It is our pleasure to acknowledge Dr. Xiangdong Zhang for writing the program for data collection. 


\section{Conflicts of Interest}

The authors declare no conflicts of interest regarding the publication of this paper.

\section{References}

[1] Han, J. and Gallagher, T.F. (2009) Physical Review A, 77, Article ID: 015404.

[2] Han, J. and Hu, C.Y. (2016) Molecular Physics, 114, 637. https://doi.org/10.1080/00268976.2015.1109150

[3] Han, J. (2017) Physical Review A, 95, Article ID: 062502.

[4] Han, J. (2017) Molecular Physics, 115, 2479.

[5] Bhaa, S.E.A. and Malvin, T.C. (1991) Fundamentals of Photonics. John Wiley \& Sons Inc., New York.

[6] Zhou, B.K. (1980) Laser Theory. National Defense Industry Press, Beijing.

[7] Wyatt, R. and Devlin, W.J. (1983) Electronic Letters, 19, 110112. https://doi.org/10.1049/el:19830079

[8] Wieman, C.E. and Hollberg, L. (1991) Review of Scientific Instruments, 62, 1-20. https://doi.org/10.1063/1.1142305

[9] MacAdam, K.B., Steinbach, A. and Wieman, C. (1992) American Journal of Physics, 60, 1098-1111. https://doi.org/10.1119/1.16955

[10] Littman, M.G. and Metcalf, H.J. (1978) Applied Optics, 17, 22242227. https://doi.org/10.1364/AO.17.002224

[11] Littman, M.G. (1978) Optics Letters, 3, 138-140. https://doi.org/10.1364/OL.3.000138

[12] Shih, M.C., Wang, S.C. and Liang, C.W. (2005) Journal of Vacuum Science \& Technology B, 23, 2180-2184. https://doi.org/10.1116/1.2009772

[13] Soletsky, P.A., Bixler, D.L. and Dunning, F.B. (1994) Review of Scientific Instruments, 65, 3585-3587. https://doi.org/10.1063/1.1144541

[14] Gallagher, T.F. (1994) Rydberg Atoms. Cambridge University Press, Cambridge. https://doi.org/10.1017/CBO9780511524530

[15] Demtroder, W. (2003) Laser Spectroscopy. 3rd Edition, SpringerVerlag, Berlin, Heidelberg, New York. https://doi.org/10.1007/978-3-662-05155-9

[16] Matthew, S.C. (2003) External Cavity Diode Lasers: Controlling Laser Output via Optical Feedback. B.S. Thesis, College of William and Mary, Williamsburg, VA.

[17] Ryan, C. (2015) Construction and Optimization of a Tapered Amplifier System for Applications in Ultra-Cold Plasma Research. Honors Thesis (Adviser Duncan Tate), Colby College, Waterville, ME. 
[18] Jun, Y. and Steven, C.T. (2005) Femtosecond Optical Frequency Comb: Principle, Operation, and Application. Springer, New York. 